\title{
Azithromycin in the Successful Management of COVID-19: A Family Physician's Perspective
}

\author{
Leonid Tafler $^{1}$, Anastasia Danilevsky ${ }^{2}$, Divya Seth ${ }^{3}$ \\ 1. Primary Care, Touro College of Osteopathic Medicine, New York, USA 2. Medicine, St. George's University, St. \\ George, GRD 3. Family Medicine, Touro College of Osteopathic Medicine, New York, USA
}

Corresponding author: Divya Seth, dseth@student.touro.edu

\begin{abstract}
The novel severe acute respiratory syndrome coronavirus 2 (SARS-COV-2), causing coronavirus disease-19 (COVID-19), has been responsible for approximately 75 million cases and 1.6 million deaths globally as of December 22, 2020. Currently, no treatment modalities or management options have been recommended by the National Institutes of Health (NIH) prior to patient hospitalization and supplemental oxygen requirement. This poses a unique challenge for outpatient primary care physicians, who are often tasked with initial care of patients early on in their disease course. During the pandemic, our family practice provided medical care to approximately 2,000 families located in the surrounding Brooklyn community. With only telemedicine at our disposal, our clinic was tasked with treating patients presenting remotely who may or may not have had COVID-19 - a large clinical diagnosis was made given the absence of in-person testing. Often co-administered, Azithromycin was considered a supportive agent that may or may not have increased the benefit of hydroxychloroquine. However, Azithromycin may perform well on its own for various reasons as it has been shown to have antiviral activity against other RNA viruses, anti-inflammatory properties, and antiviral effects within bronchial epithelial cells. Azithromycin has also shown efficacy as an add-on treatment for reducing asthma exacerbations - pertinent to the pro-inflammatory pulmonary conditions in COVID-19 progression - and may even prevent or treat bacterial co-infection in patients with
\end{abstract} SARS-COV-2.

In order to investigate the association between Azithromycin and the COVID-19 disease process, our clinical study retrospectively identified patients who were prescribed Azithromycin (500 mg on day one $+250 \mathrm{mg}$ on days two to five) during the peak months of the COVID-19 pandemic in New York City from March 2020 through May 2020. All patients prescribed Azithromycin with suspicion of COVID-19 infection were interviewed via telephone regarding their constellation of symptoms, compliance with the prescribed antibiotic for the intended course, symptom duration prior to and following antibiotic course initiation, as well as any further complications of their illness, if present. Ultimately, the majority of the patients who were interviewed over the phone concluded that a full course of Azithromycin helped improve their symptoms during their infection with COVID-19. Outcomes and complications in patients treated with Azithromycin were noteworthy in that there were no reports of pulmonary complications or deterioration of pulmonary function after treatment (e.g., no shortness of breath, wheezing, dyspnea, etc.), although some patients did experience residual coughing and nasal discharge post-treatment. We believe further study of this treatment in the setting of experimental, randomized controlled trials may reveal the benefits of Azithromycin in terms of reducing infection severity, length, and limiting the incidence of complications in patients with COVID-19.

Categories: Family/General Practice, Infectious Disease, Public Health

Keywords: covid-19 outbreak, azithromycin, critical care, epidemiology, hospitalization, sars-cov-2, antiviral, supportive therapy, ambulatory treatment

\section{Introduction}

The novel severe acute respiratory syndrome coronavirus 2 (SARS-COV-2), causing coronavirus disease-19 (COVID-19), has been responsible for approximately 75 million cases and 1.6 million deaths globally as of December 22, 2020 [1]. Antecedent to patient hospitalization for COVID-19, no treatment modalities are currently recommended by the National Institutes of Health (NIH) for outpatient use [2]. This poses a unique challenge for outpatient primary care physicians, who are often tasked with initial care of patients early on in their disease course [3]. During the early stages of the pandemic in the United States, "stay-at-home" orders and social distancing requirements changed the nature of initial patient care from in-person to virtual telehealth visits [4]. Duke University School of Medicine reported an increase in their share of telehealth visits from $<1 \%$ to $70 \%$ of visits in four weeks [4]. During this time, family physicians have been advised to screen their patients virtually for COVID-19 "red flags" (symptoms indicating moderate to severe disease), provide testing recommendations, counsel their patients regarding isolation precautions if disease is suspected, and provide supportive management [5,6]. During the pandemic, our family practice provided medical care to approximately 2,000 families located in the surrounding Brooklyn community. With only telemedicine at our disposal, our clinic was tasked with treating patients presenting remotely who may or may not have had COVID-19 - a large clinical diagnosis was made, given the absence of in-person testing. 
However, no treatments have been recommended for COVID-19 outpatient use nor for the early stages of the disease, leaving family practices like ours puzzled by how best to manage patients in the midst of a pandemic [5].

It has been proposed that viral loads in COVID-19 patients are highest at the time of symptom onset and decline thereafter; thus, in the event that COVID-19 is suspected at first contact with a primary care physician, it may be beneficial to explore pharmacologic therapy for early management of COVID-19 [6,7]. Several agents are currently being studied for this purpose. Among them, hydroxychloroquine was proposed to have properties that could aid COVID-19 treatment: interfering with viral replication, fever suppression, and immunomodulatory effects [8]. However, subsequent studies have revealed that there is not enough evidence for or against this agent in the treatment of COVID-19 [5]. Other treatment modalities being studied for early/outpatient use include various antiviral drugs, monoclonal antibodies, systemic corticosteroids, and some antibiotics (namely Azithromycin) [6,9]. Of these therapies, Azithromycin has been the treatment most commonly prescribed for COVID-19 in the outpatient setting and is associated with a well-tolerated adverse effect profile [10]. Often co-administered, Azithromycin was considered a supportive agent that may or may not have modulated the effects of hydroxychloroquine [10]. Ultimately, subsequent studies revealed that there is not enough evidence for or against the use of hydroxychloroquine in COVID-19; however, Azithromycin may perform well on its own for various reasons. It has been shown to have antiviral activity against other RNA viruses, anti-inflammatory properties, and antiviral effects within bronchial epithelial cells [9,11-13]. Azithromycin has also shown efficacy as an add-on treatment for reducing asthma exacerbations - pertinent to the pro-inflammatory pulmonary conditions in COVID-19 progression - and may even prevent or treat bacterial co-infection in patients with SARS-COV-2 [9,14].

In this observational study, we present the outcomes of patients with strong clinical suspicion of COVID-19 who presented to our family practice remotely for care. With no better treatment option available, patients were treated with Azithromycin, in addition to being given all recommended guidelines for self-isolation and testing, with strongly positive clinical responses and few complications.

\section{Materials And Methods}

In order to investigate the association between Azithromycin and the COVID-19 disease process, our clinical study identified patients who were prescribed Azithromycin ( $500 \mathrm{mg}$ on day one $+250 \mathrm{mg}$ on days two to five) during the peak months of the COVID-19 pandemic in New York City from March 2020 through May 2020. Patient information was collected through NextGen Office, the electronic medical record (EMR) being used by our clinic. Inclusion criteria for patients comprised an electronic medical record (EMR) record of Azithromycin prescription between March 23, 2020 and May 31, 2020 (n = 39). Once patients who fit the inclusion criteria were compiled, additional information was gathered, including the date Azithromycin was prescribed, patient history of exposure to COVID-19, history of COVID-19 antibody test, history of COVID19 antigen test, and history of COVID-19 symptoms (e.g., shortness of breath, cough, mild fever, loss of taste/smell +/- abdominal complaints). Once patient information was compiled, patients were contacted to follow up on their symptoms. All patients prescribed Azithromycin with clinical suspicion of COVID-19 infection were interviewed via telephone regarding their constellation of symptoms, compliance with the prescribed antibiotic for the intended course, symptom duration prior to and following antibiotic course initiation, as well as any further complications of their illness, if present. Patients who were included in the study were not prescribed any other medication alongside Azithromycin, other than non-steroidal antiinflammatory drugs (NSAIDs) or acetaminophen as supportive therapies. During the initial encounter, all patients were advised to self-quarantine at home under suspicion of being contagious with COVID-19.

\section{Results}

Of the 39 patients who were prescribed Azithromycin, 18 had also tested positive for COVID-19 antibodies at a follow-up visit. We deemed these patients likely to be COVID-19 positive at their initial telehealth visit based on their clinical presentation and their subsequent antibody results, and grouped them together as Group A. Each of these patients were contacted individually and interviewed over the phone. Patients were asked a number of preset questions (Figure 1), and their responses were recorded as shown in Table 1. Of the patients in Group A, 12 stated that the Azithromycin course decreased the intensity of their symptoms within one week, four stated that their symptoms persisted for exactly one week, and two patients reported their symptoms persisted for more than one week. Sixteen patients $(n=16)$ within Group A presented to their telemedicine appointment at the initiation of their symptoms, while two patients $(\mathrm{n}=2)(\# 15$ and \#39) presented after symptoms had worsened. 


\section{Cureus}

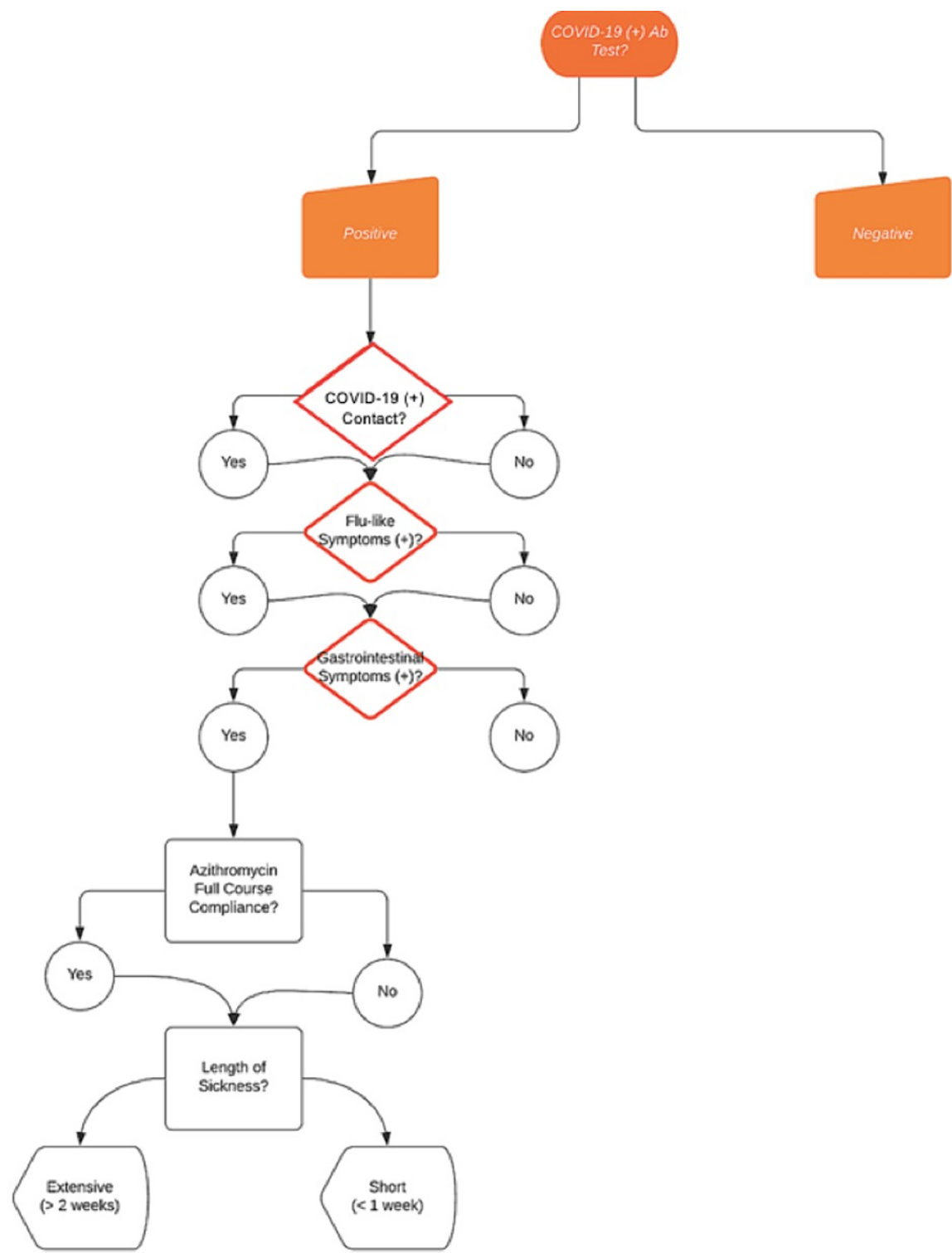

FIGURE 1: Preset questions flowchart

\begin{tabular}{|c|c|c|c|c|c|c|}
\hline Patient \# & Contact with COVID-19 (+)? & Flu-like symptoms? & Gl symptoms? & Azithromycin compliance? & Length of sickness post-treatment? & Complications? \\
\hline 1 & No & Yes & No & Yes & 2 days & Abdominal pain \\
\hline 2 & No & Yes & No & No & 1 day & None \\
\hline 3 & Yes & Yes & Yes & Yes & 5 days & Slight cough \\
\hline 4 & No & Yes & Yes & Yes & Few days & None \\
\hline 5 & Yes & No & Yes & Yes & $>1$ week & None \\
\hline 6 & No & Yes & No & Yes & Few days & None \\
\hline 7 & Yes & Yes & Yes & Yes & 4 days & Slight cough \\
\hline 8 & No & No & Yes & Yes & 1 week & None \\
\hline 9 & No & Yes & No & Yes & Few days & None \\
\hline 10 & No & No & No & No & None & None \\
\hline 11 & No & Yes & No & Yes & Few days & Abdominal pain \\
\hline
\end{tabular}




\section{Cureus}

\begin{tabular}{|c|c|c|c|c|c|c|}
\hline 12 & Yes & Yes & No & Yes & 1 week & Runny nose \\
\hline 13 & No & Yes & Yes & Yes & Few days & None \\
\hline 14 & No & No & No & Yes & 1 day & None \\
\hline 15 & Yes & Yes & Yes & Yes & Few days & Slight cough \\
\hline 16 & No & Yes & Yes & Yes & 5 days & None \\
\hline 17 & Yes & Yes & No & Yes & $<1$ week & None \\
\hline 18 & Yes & Yes & Yes & Yes & 4 days & Runny nose \\
\hline 19 & Yes & No & Yes & Yes & Few days & Postnasal drip \\
\hline 20 & No & Yes & No & Yes & 2 days & None \\
\hline 21 & No & Yes & No & Yes & 3 days & Diarnea \\
\hline 22 & Yes & Yes & Yes & Yes & <1 week & Slight cough \\
\hline${ }^{23}$ & Yes & Yes & Yes & Yes & Few days & Slight cough \\
\hline${ }^{24}$ & No & No & Yes & Yes & 2 days & None \\
\hline 25 & No & Yes & No & Yes & 1 day & None \\
\hline 26 & Yes & Yes & Yes & Yes & Few days & Runny nose \\
\hline 27 & Yes & Yes & Yes & Yes & $>1$ week & None \\
\hline 28 & No & Yes & No & № & Few days & None \\
\hline${ }^{29}$ & No & No & Yes & Yes & 2 days & None \\
\hline 30 & No & Yes & No & Yes & 2 days & None \\
\hline 31 & No & Yes & No & Yes & 1 day & None \\
\hline 32 & Yes & Yes & Yes & Yes & Few days & None \\
\hline${ }^{33}$ & No & Yes & № & Yes & Few days & None \\
\hline${ }^{34}$ & Yes & Yes & No & Yes & Few days & None \\
\hline 35 & No & Yes & No & Yes & <1 week & None \\
\hline${ }^{36}$ & No & No & Yes & № & 3 days & None \\
\hline 37 & Yes & Yes & No & Yes & 1 day & None \\
\hline${ }^{38}$ & Yes & Yes & Yes & Yes & 1 week & None \\
\hline 39 & Yes & Yes & No & Yes & 1 week & Nor \\
\hline
\end{tabular}

\section{TABLE 1: Patient response record}

GI, Gastrointestinal.

Patients who were prescribed Azithromycin during the peak of the pandemic with unknown COVID-19 antibody status were referred to as Group B. Some of the patients were able to provide us with further information of their COVID-19 antibody testing status, which was positive, however, unconfirmed by the clinic. The rest of the patients were asked regarding their symptoms during the peak months of pandemic and whether they were tested for antibodies or whether they believed that they were sick with COVID-19. Some of the patients were tested and results were negative, and some patients believed that they were infected with COVID-19 but were never tested during or after their sickness (Table 2).

\begin{tabular}{|c|c|c|c|c|}
\hline Patient & Azithromycin Prescription Date & COVID Antibody Test Date & Time Between & CoVID Antibody Test Results \\
\hline 1 & $3 / 25 / 2020$ & $5 / 20 / 2020$ & 56 & Negative \\
\hline 2 & $3 / 23 / 2020$ & $4 / 28 / 2020$ & 36 & Negative \\
\hline
\end{tabular}




\section{Cureus}

\begin{tabular}{|c|c|c|c|c|}
\hline 3 & $3 / 24 / 2020$ & $8 / 11 / 2020$ & 140 & Positive \\
\hline 4 & $4 / 2 / 2020$ & 6/29/2020 & 88 & Positive \\
\hline 5 & $3 / 24 / 2020$ & $8 / 11 / 2020$ & 140 & Positive \\
\hline 6 & $3 / 27 / 2020$ & $5 / 22 / 2020$ & 56 & Negative \\
\hline 7 & $3 / 25 / 2020$ & $5 / 9 / 2020$ & 45 & Positive \\
\hline 8 & $3 / 25 / 2020$ & 9/11/2020 & 170 & Positive \\
\hline 9 & $3 / 25 / 2020$ & $5 / 26 / 2020$ & 62 & Negative \\
\hline 10 & $3 / 30 / 2020$ & 4/24/2020 & 25 & Negative \\
\hline 11 & $3 / 25 / 2020$ & $4 / 27 / 2020$ & 33 & Negative \\
\hline 12 & $4 / 1 / 2020$ & $5 / 13 / 2020$ & 42 & Positive \\
\hline 13 & $3 / 26 / 2020$ & $5 / 2 / 2020$ & 37 & Negative \\
\hline 14 & $3 / 27 / 2020$ & $5 / 22 / 2020$ & 56 & Negative \\
\hline 15 & $4 / 2 / 2020$ & $4 / 20 / 2020$ & 18 & Positive \\
\hline 16 & $3 / 30 / 2020$ & 9/22/2020 & 176 & Positive \\
\hline 17 & $3 / 31 / 2020$ & 10/9/2020 & 192 & Positive \\
\hline 18 & $4 / 1 / 2020$ & $5 / 20 / 2020$ & 49 & Positive \\
\hline 19 & 4/2/2020 & $4 / 22 / 2020$ & 40 & Positive \\
\hline 20 & $4 / 3 / 2020$ & $6 / 1 / 2020$ & 20 & Negative \\
\hline 21 & $4 / 6 / 2020$ & 10/21/2020 & 59 & Negative \\
\hline 22 & $4 / 14 / 2020$ & 10/24/2020 & 198 & Positive \\
\hline 23 & $4 / 6 / 2020$ & $7 / 8 / 2020$ & 193 & Positive \\
\hline 24 & $4 / 6 / 2020$ & $6 / 5 / 2020$ & 93 & Negative \\
\hline 25 & $4 / 8 / 2020$ & $4 / 29 / 2020$ & 60 & Negative \\
\hline 26 & $4 / 8 / 2020$ & $6 / 30 / 2020$ & 21 & Positive \\
\hline 27 & $4 / 8 / 2020$ & 6/30/2020 & 83 & Positive \\
\hline 28 & $5 / 13 / 2020$ & $7 / 8 / 2020$ & 56 & Negative \\
\hline 29 & $4 / 9 / 2020$ & 8/30/2020 & 82 & Negative \\
\hline 30 & $4 / 10 / 2020$ & $5 / 4 / 2020$ & 24 & Negative \\
\hline 31 & $4 / 13 / 2020$ & $4 / 30 / 2020$ & 17 & Negative \\
\hline 32 & $4 / 15 / 2020$ & $7 / 12 / 2020$ & 88 & Positive \\
\hline 33 & $4 / 20 / 2020$ & $5 / 4 / 2020$ & 14 & Negative \\
\hline 34 & $4 / 22 / 2020$ & $4 / 22 / 2020$ & 0 & Negative \\
\hline 35 & $5 / 4 / 2020$ & $5 / 4 / 2020$ & 0 & Negative \\
\hline 36 & $5 / 1 / 2020$ & $4 / 30 / 2020$ & -1 & Negative \\
\hline 37 & $4 / 30 / 2020$ & $4 / 30 / 2020$ & 0 & Negative \\
\hline 38 & $5 / 13 / 2020$ & $4 / 22 / 2020$ & 21 & Positive \\
\hline 39 & $4 / 25 / 2020$ & $6 / 27 / 2020$ & 45 & Positiv \\
\hline
\end{tabular}

TABLE 2: Azithromycin original table 


\section{Discussion}

Interest in this observational research study originated from the difficulties our practice faced in treating patients with a high clinical suspicion of COVID-19 via telemedicine. During the peak of the pandemic, many patients sought care from their primary care physicians and presented remotely with new-onset COVID-like symptoms. However, two of our patients presented to our clinic after developing symptoms that had led to a visit to the Emergency Department. In the Emergency Department, these patients were tested for COVID-19 and were instructed to quarantine with supportive therapy and fever suppressants. These patients were then contacted by the Emergency Department within a few days with news of a positive COVID-19 test result and were instructed to continue self-quarantining at home for the next 14 days. During this time, quarantined patients were divided between those who recovered versus those whose condition worsened, requiring further hospitalization and, in some cases, ventilatory support.

One of the patients interviewed from Group A, Patient \#15, presented with this particular pattern of disease course. Patient \#15 originally presented to her local Emergency Department with COVID-like symptoms and was promptly tested for COVID-19. After being tested, the patient was advised to return home, self-isolate, and wait for the results. The patient stated that she did as she was advised, and after two days, she was contacted by the hospital and notified that she tested positive for COVID-19. She was mandated to stay home and self-quarantine for 14 days. The patient was advised to stay on bed rest during this time period and to maintain supportive care. During the quarantine period, the patient's symptoms continued to worsen until the patient decided to reach out to her primary care physician at our clinic for a telemedicine appointment. At the time of her visit, the patient's condition had deteriorated: She described severe shortness of breath, difficulty taking deep breaths, and a severe cough. Fearing secondary opportunistic bacterial infection and further deterioration of the patient's condition, the patient was prescribed Azithromycin. When interviewed, the patient stated that she felt better within days of taking the antibiotic. The patient was able to complete the full course of antibiotics and was under the impression that if not for the antibiotic, she would have been ventilated within a matter of days.

Encouraged, our clinic's treating physician continued to prescribe Azithromycin, weighing its benefits (e.g., treating opportunistic bacterial infections, reducing chronic respiratory tract disease exacerbations, and its potential antiviral and immunologic properties) against its drawbacks (e.g., the patient's potential for antibiotic resistance, Azithromycin's adverse effect profile). Social distancing and supportive therapies aside, there were no other outpatient guidelines during the peak of the COVID-19 pandemic; thus, the encouraging history of Azithromycin use (as detailed previously) did not hold insignificant weight in treating our patients with no other recourse and worsening COVID-19 symptoms. Ultimately, the majority of the patients who were interviewed over the phone concluded that a full course of Azithromycin helped improve their symptoms during their infection with COVID-19. Outcomes and complications in patients treated with Azithromycin were noteworthy in that there were no reports of pulmonary complications or deterioration of pulmonary function after treatment (e.g., no shortness of breath, wheezing, dyspnea, etc.), although some patients did experience residual coughing and nasal discharge post-treatment.

While the authors do feel that, among our sample patient population, Azithromycin may have decreased the severity of COVID-19 symptoms and/or reduced the longevity of the disease process, this study does have several limitations to address. Our sample population does not have enough power to represent the entirety of New York City's COVID-19 patient population, as our clinic did not have the access to reach many numbers of patients during the pandemic. Further, this study does not demonstrate definitively that Azithromycin is an effective treatment for COVID-19 as the study is structured as an observational study and cannot determine causative relationships, only correlative ones. Finally, we cannot definitively claim that our patient sample did indeed have COVID-19 prior to being prescribed Azithromycin rather than afterward as we were unable to conduct COVID-19 tests on initial presentation due to pandemic restrictions. Our conclusions are thus based on strong clinical suspicion that patients presented with COVID-19 due to clinical presentation and COVID-19 antibody results upon follow-up.

\section{Conclusions}

This observational study was conducted to demonstrate the efficacy of Azithromycin in treating COVID-19 from the outpatient perspective of a primary care provider. Especially when prescribed at the onset of patient symptoms, Azithromycin was well-tolerated, and the patients demonstrated improvement of symptoms within seven days. In many cases, Azithromycin use was even correlated with reversing the deterioration of patients who reported worsening of their symptoms in quarantine.

Due to the novel nature of this pandemic, our reliance on telemedicine, and the observational, retrospective structure of this study, we acknowledge that our options for patient randomization, control groups, and blinding were limited. However, the patients we treated with Azithromycin reported no adverse effects, and there were no cases of decompensation or further deterioration in any of our patients. Thus, we believe our observations may be of use in opening doors for further discussion and potential use of Azithromycin in the outpatient setting during symptom onset in patients with suspected COVID-19 infection. We believe further study of this treatment in the setting of experimental, randomized controlled trials may reveal the benefits 
of Azithromycin in terms of reducing infection severity, length, and limiting the incidence of complications in patients with COVID-19.

\section{Additional Information \\ Disclosures}

Human subjects: Consent was obtained or waived by all participants in this study. Animal subjects: All authors have confirmed that this study did not involve animal subjects or tissue. Conflicts of interest: In compliance with the ICMJE uniform disclosure form, all authors declare the following: Payment/services info: All authors have declared that no financial support was received from any organization for the submitted work. Financial relationships: All authors have declared that they have no financial relationships at present or within the previous three years with any organizations that might have an interest in the submitted work. Other relationships: All authors have declared that there are no other relationships or activities that could appear to have influenced the submitted work.

\section{References}

1. Weekly epidemiological update - 22 December . (2020). Accessed: December 24, 2020: https://www.who.int/publications/m/item/weekly-epidemiological-update---22-december-2020.

2. Therapeutic management of patients with COVID-19. (2020). Accessed: December 17, 2020: https://www.covid19treatmentguidelines.nih.gov/therapeutic-management/..

3. Rawaf S, Allen LN, Stigler FL, Kringos D, Yamamoto HQ, van Weel C: Lessons on the COVID-19 pandemic, for and by primary care professionals worldwide. Eur J Gen Pract. 2020, 26:129-33. 10.1080/13814788.2020.1820479

4. Wosik J, Fudim M, Cameron B, et al.: Telehealth transformation: COVID-19 and the rise of virtual care . J Am Med Inform Assoc. 2020, 27:957-62. 10.1093/jamia/ocaa067

5. Cheng A, Caruso D, McDougall C: Outpatient management of COVID-19: rapid evidence review. Am Fam Physician. 2020, 102:478-86.

6. Lotfi M, Hamblin MR, Rezaei N: COVID-19: transmission, prevention, and potential therapeutic opportunities. Clin Chim Acta. 2020, 508:254-66. 10.1016/j.cca.2020.05.044

7. Walsh KA, Jordan K, Clyne B, et al.: SARS-CoV-2 detection, viral load and infectivity over the course of an infection. J Infect. 2020, 81:357-71. 10.1016/j.jinf.2020.06.067

8. Bhandari R, Khanna G, Kuhad A: Pharmacological insight into potential therapeutic agents for the deadly Covid-19 pandemic. Eur J Pharmacol. 2021, 890:173643. 10.1016/j.ejphar.2020.173643

9. Echeverría-Esnal D, Martin-Ontiyuelo C, Navarrete-Rouco ME, De-Antonio Cuscó M, Ferrández O, Horcajada JP, Grau S: Azithromycin in the treatment of COVID-19: a review . Expert Rev Anti Infect Ther. 2021, 19:147-63. 10.1080/14787210.2020.1813024

10. Oldenburg CE, Doan T: Azithromycin for severe COVID-19. Lancet. 2020, 396:936-7. 10.1016/S01406736(20)31863-8

11. Li C, Zu S, Deng YQ, et al.: Azithromycin protects against Zika virus Infection by upregulating virus-induced type I and III interferon responses [IN PRESS]. Antimicrob Agents Chemother. 2019, 10.1128/AAC.00394-19

12. Menzel M, Akbarshahi H, Bjermer L, Uller L: Azithromycin induces anti-viral effects in cultured bronchial epithelial cells from COPD patients. Sci Rep. 2016, 6:28698. 10.1038/srep28698

13. Bleyzac N, Goutelle S, Bourguignon L, Tod M: Azithromycin for COVID-19: more than just an antimicrobial?. Clin Drug Investig. 2020, 40:683-6. 10.1007/s40261-020-00933-3

14. Gibson PG, Yang IA, Upham JW, et al.: Effect of azithromycin on asthma exacerbations and quality of life in adults with persistent uncontrolled asthma (AMAZES): a randomised, double-blind, placebo-controlled trial. Lancet. 2017, 390:659-68. 10.1016/S0140-6736(17)31281-3 